\title{
Geração e transmissão da energia elétrica: impacto sobre os povos indígenas no Brasil
}

\author{
Electric power generation and transmission: \\ the impact on indigenous peoples in Brazil
}

Sergio Koifman 1

\footnotetext{
1 Departamento de Epidemiologia e Métodos Quantitativos em Saúde, Escola Nacional de Saúde Pública, Fundação Oswaldo Cruz.

Rua Leopoldo Bulhões 1480, Rio de Janeiro, $R J$ 21041-210, Brasil. koifman@ensp.fiocruz.br
}

\begin{abstract}
This paper presents an overview of the effects of electric power generation and transmission on indigenous communities in Brazil. According to data from FUNAI (the Brazilian government's Board of Indian Affairs), there are 156 cases of direct impact, present or future, of the electric power sector on Indian settlements geographically distributed throughout Brazil, 65\% of which are located in the Northern Region of the country. The principal complaints by indigenous communities relate to the direct effects of flooding following construction of hydroelectric dams, destruction of sacred sites like cemeteries, mosquito proliferation, and health-related hazards such as malaria and other infectious diseases, decrease in game for hunting, crowding out of farm land, and increased invasion of indigenous lands. Future perspectives include a scenario with further construction of hydroelectric dams, especially in the Amazon region, with possible similar effects on indigenous communities.
\end{abstract}

Key words Electricity; Environmental Impact; South American Indians

Resumo Este trabalho busca retratar a distribuição de intercorrências nas comunidades indígenas no Brasil associadas à geração e à distribuição da energia elétrica. De acordo com dados da Fundação Nacional do Índio, há 156 áreas geograficamente distribuídas no país caracterizadas por afetarem, presente ou futuramente, assentamentos indígenas através da expansão do setor elétrico - 65\% das quais situadas na Região Norte. As principais reclamações destas comunidades dizem respeito aos efeitos diretos acarretados pelo alagamento originado com a construção das barragens hidroelétricas: submersão de territórios sagrados (como cemitérios); proliferação de mosquitos (ampliando a difusão da malária e de outras doenças infecciosas); escassez de caça; restrição das terras para a agricultura; e a criação de condições facilitadoras da invasão de terras indígenas. O cenário de perspectivas futuras é esboçado em quadro marcado pelo planejamento da construção de novas usinas hidrelétricas - em especial, na região amazônica - com possiveis efeitos similares nas comunidades indígenas.

Palavras-chave Eletricidade; Impacto Ambiental; Índios Sul-Americanos 
A expansão do setor elétrico no Brasil tem acarretado historicamente situações de conflito direto ou latente com diversas comunidades indígenas. Este fato tem ocorrido sobretudo em decorrência das conseqüências advindas da construção de usinas hidrelétricas e de linhas de transmissão de alta tensão elétrica no interior ou nas proximidades das terras indígenas.

Entre as principais interferências diretas e indiretas oriundas pela expansão do setor elétrico nas áreas indígenas, destacam-se: a realocação de comunidades para outras regiões, muitas vezes acompanhada de rupturas em seus estilos de vida; o alagamento de grandes parcelas territoriais, incluindo áreas consideradas sagradas, como os locais tradicionais de sepultamento; a invasão das terras indígenas, facilitadas indiretamente pela expansão das instalações do setor elétrico; a diminuição da disponibilidade da caça e a redução das áreas cultiváveis; e a proliferação da população de vetores, incluindo artrópodos e moluscos, levando ao aumento na incidência de malária e outras doenças infecciosas (Arruda, 1985; Bulcão, 1994; Consolini et al., 1990; Couto, 1996; Guimarães et al., 1997; Tadei, 1994).

Relatos desta natureza têm sido feitos por diferentes comunidades, conforme apresentado na Tabela 1. Alguns exemplos poderiam ser citados, como os Avá-Guaraní, em decorrência da construção da Usina Itaipu-Binacional; os Parakanã, após a construção da Usina de Tucuruí; os Pankararu, com o funcionamento da Usina Luiz Gonzaga. A construção de linhas de transmissão de alta tensão elétrica no interior das terras indígenas tem sido outro importante fator de litígio, como os Parakatejé, no Pará; os Pankararu, em Pernambuco; os Nambikwára, em Mato Grosso, e os Kaigáng, no Paraná, entre outras comunidades. Embora estas tenham sido construídas após a assinatura de acordos, seja com o pagamento de indenizações e/ou instalação da rede elétrica nos domicílios das aldeias, o sentimento de não aceitação de seus termos persiste em diferentes comunidades (Ferraz, comunicação pessoal).

Neste trabalho, procuraremos dimensionar a magnitude das interferências em diversas comunidades indígenas acarretadas pela expansão do setor elétrico no país. Antes de retratála, contudo, é oportuno caracterizar as diferentes etapas envolvidas no processo de construção de uma usina hidrelétrica, desde a de planejamento até a sua entrada em operação.

De acordo com as Centrais Elétricas Brasileiras S. A. (ELETROBRÁS, 1990), a primeira etapa diz respeito ao inventário da bacia hidrográfica, quando são realizados estudos a res- peito de seu potencial, de como a eletricidade gerada poderá ser aproveitada, bem como são analisadas as opções para a localização da barragem. Segue-se a fase de estudos de viabilidade, em que há análise de casos específicos e da relação custo-benefício de cada projeto. Estas geram o desenho de um Projeto Básico, que se constitui na última etapa dos estudos, subsidiando as decisões do setor elétrico e incluindo os impactos negativos acarretados pela obra. Passa-se então à etapa do Projeto Executivo, quando se inicia a construção da barragem e, com esta, a execução das atividades de desmatamento e alteração do curso fluvial, acelerando o processo migratório para a área. Finalmente, após seu término, inicia-se a etapa de operação com a geração e distribuição da energia elétrica através das linhas de transmissão de alta tensão, quando surgem os riscos decorrentes da formação dos grandes lagos e proliferação de vetores.

Segundo dados fornecidos pela Fundação Nacional do Índio (FUNAI) em 1996 (comunicação pessoal), existiam 156 Áreas Indígenas com algum tipo de contestação em decorrência de litígios com empresas do setor elétrico (Tabela 1). Estas apresentavam a seguinte distribuição geográfica: 39 no Estado do Amazonas; 22 em Mato Grosso; 21 no Pará; 14 em Mato Grosso do Sul; 13 em Rondônia; oito no Rio Grande do Sul; sete no Paraná; seis em Tocantins; quatro cada em Pernambuco, Bahia e Santa Catarina; três em Minas Gerais; dois cada em Alagoas e Maranhão; e um em cada um dos estados de Goiás, Espirito Santo, Paraíba, Roraima, Sergipe, São Paulo e Amapá. Percebe-se assim não só um padrão nacional de distribuição da problemática, mas também sua concentração na Região Norte, com mais de $65 \%$ dos litígios referidos pelas comunidades indígenas com o setor elétrico (FUNAI, 1996, comunicação pessoal).

Em conjunto, as populações destas comunidades capazes de virem a ser afetadas direta ou potencialmente pela expansão deste setor totalizam ao redor de 117.837 indígenas segundo estatísticas da FUNAI, aproximadamente um terço de toda a população indígena no país (MS, 2000). Neste sentido, o principal fator envolvido diz respeito à construção de usinas hidrelétricas, respondendo por 104 (67\%) das interferências documentadas com áreas indígenas, estando 26 destas em operação. A segunda causa mais freqüente de interferência foi a implantação de linhas de transmissão de alta tensão elétrica no interior das terras indígenas (26 relatos), seguindo-se cinco relatos referentes à proximidade destas com áreas limítrofes à comunidades indígenas. 
Modalidades de interferência do setor elétrico em áreas indígenas no Brasil, 1996.

\begin{tabular}{|c|c|c|c|c|c|}
\hline Área indígena & Estado & Superficie $\left(\mathrm{km}^{2}\right)$ & População & Grupo indígena & Tipo de interferência \\
\hline Aldeia Campestre & MS & 9 & 223 & Guaraní-Kaiwá & LT limítrofe com Al com ramificação até a aldeia. \\
\hline Aldeia Limão Verde & MS & 668 & 390 & Guaraní-Kaiwá & LT de $13,8 \mathrm{kv}$ cruzando $2 \mathrm{~km}$ da Al. \\
\hline Amambai & MS & 2.429 & 4.535 & Guaraní-Kaiwá & LT de $13,8 \mathrm{kv}$ com extensão de $3 \mathrm{~km}$ na Al. \\
\hline Andirá Marau & AM & 788.528 & 4.890 & Sateré-Mawé & UHE Itaituba planejada. \\
\hline Apinajé & TO & 141.904 & 904 & Apinayé & $\begin{array}{l}\text { UHE Santo Antônio do Tocantins planejada; } \\
\text { UHE Santa Izabel planejada; UHE Serra Quebrada } \\
\text { planejada; LT trecho Imperatriz/Tocantinópolis } \\
\text { cruzando Al. }\end{array}$ \\
\hline Apucarana & PR & 5.574 & 509 & Kaingáng & $\begin{array}{l}\text { UHE São Jerônimo planejada; UHE Apucaraninha } \\
\text { em operação. Impacto direto. }\end{array}$ \\
\hline Apyterewa & PA & 980.000 & 186 & Parakanã & UHE Complexo do Xingu planejada. \\
\hline Arára & PA & 301.600 & 82 & Arára & UHE Complexo do Xingu planejada. \\
\hline $\begin{array}{l}\text { Araweté Igarapé } \\
\text { Ipixuna }\end{array}$ & PA & 985.000 & 226 & Araweté & UHE Complexo do Xingu planejada. \\
\hline Areões & MT & 218.515 & 522 & Xavánte & $\begin{array}{l}\text { UHE Torixoréu planejada; Estudo de viabilidade } \\
\text { para UHE Barra do Peixe, UHE Couto Magalhães } \\
\text { e UHE Foz do Nodoire. }\end{array}$ \\
\hline Avá-Canoeiro & GO & 38.000 & 5 & Avá-Canoeiro & $\begin{array}{l}\text { UHE Serra da Mesa em construção; UHE Cana } \\
\text { Brava/FURNAS com estudo de viabilidade; } \\
\text { UHE Mirador planejada. }\end{array}$ \\
\hline Avá-Guaraní Ocoi & PR & 232 & 215 & Avá-Guaraní & $\begin{array}{l}\text { UHE Itaipu-Binacional acarretou transferência } \\
\text { da comunidade para área de } 232 \mathrm{ha} \text {, considerada } \\
\text { insuficiente, às margens do lago. Impacto ambiental: } \\
\text { desmatamento, malária, alteração na qualidade } \\
\text { da água e perda de sítios arqueológicos. }\end{array}$ \\
\hline Bacajá & PA & 192.126 & 218 & Xikrín & UHE Complexo do Xingu planejada. \\
\hline Balaio & AM & 54.840 & 200 & $\begin{array}{l}\text { Desána, Tukáno, } \\
\text { outros }\end{array}$ & UHE São Gabriel planejada. \\
\hline $\begin{array}{l}\text { Caiçara Igarapé } \\
\text { São Pedro }\end{array}$ & SE & 4.316 & 206 & Xoko & UHE Oroco e UHE Ibó planejadas. \\
\hline Caieiras Velhas & ES & 1.519 & 1.017 & $\begin{array}{l}\text { Guaraní } \\
\text { e Tupiniquim }\end{array}$ & $\begin{array}{l}\text { LT de } 13,8 \mathrm{kv} \text { no trecho Aracruz/Coqueiral acompanha } \\
\text { a estrada e cruza Al numa extensão de } 8 \mathrm{~km} \text {. }\end{array}$ \\
\hline Cambiowa & PE & 27.250 & 1.108 & Cambiowa & LT com extensão de $7 \mathrm{~km}$. \\
\hline Cana Brava Guajajára & MA & 137.329 & 3.143 & Guajajára & LT no trecho Imperatriz/Presidente Dutra. \\
\hline Capivara & AM & 650 & 140 & Múra & $\begin{array}{l}\text { UHE Monte Cristo planejada; UHE Santo Antônio, } \\
\text { UHE São Jirau e UHE Madeira Bin planejadas. }\end{array}$ \\
\hline Capoto Jarina & MT & 634.915 & 127 & Kayapó e outros & UHE Kokraimôro e UHE Jarina planejadas. \\
\hline Caramuru Paraguassu & BA & 36.000 & 1.449 & Pataxó & $\begin{array}{l}\text { LT no trecho Pau Brasil//taipu cortando } \mathrm{Al} \\
\text { numa extensão de } 2 \mathrm{~km} \text {. }\end{array}$ \\
\hline Carreteiro & RS & 602 & 202 & Kaingáng & $\begin{array}{l}\text { LT no trecho Água Santa/Linha Fernandez cortando a } \\
\text { Al numa extensão de } 2 \mathrm{~km} \text { e segue paralela a estrada. }\end{array}$ \\
\hline Cerrito & MS & 2.040 & 180 & Guaraní Nhandéva & UHE Ilha Grande planejada. \\
\hline Coata Laranjal & AM & 805.000 & 1.760 & $\begin{array}{l}\text { Mundurukú, } \\
\text { Sateré-Mawé }\end{array}$ & $\begin{array}{l}\text { UHE São Jirau; UHE Monte Cristo; UHE } \\
\text { Santo Antônio e UHE Madeira Bin planejadas. }\end{array}$ \\
\hline Cubate & AM & 23.201 & 250 & $\begin{array}{l}\text { Baníwa, Baré, } \\
\text { Kobewa }\end{array}$ & UHE São Gabriel planejada. \\
\hline
\end{tabular}

(continua) 
Tabela 1 (continuação)

\begin{tabular}{|c|c|c|c|c|c|}
\hline Área indígena & Estado & Superficie $\left(\mathrm{km}^{2}\right)$ & População & Grupo indígena & Tipo de interferência \\
\hline Cuia & AM & 1.322 & 40 & Múra & $\begin{array}{l}\text { UHE São Jirau, UHE Santo Antônio, UHE Madeira } \\
\text { Bin e UHE Monte Cristo planejadas. }\end{array}$ \\
\hline Cuiari & AM & 13.883 & 192 & $\begin{array}{l}\text { Baníwa, Baré, } \\
\text { Kobewa }\end{array}$ & UHE São Gabriel planejada. \\
\hline Cuminapanema & PA & a definir & 0 & Isolados & UHE Aparaí planejada. \\
\hline Cunhã & AM & 12.730 & 140 & Múra & $\begin{array}{l}\text { UHE Jirau, UHE Santo Antônio, UHE Madeira Bin } \\
\text { e UHE Monte Cristo planejadas. }\end{array}$ \\
\hline Dourados & MS & 3.475 & 8.971 & $\begin{array}{l}\text { Guaraní-Kaiwá, } \\
\text { Teréna }\end{array}$ & $\begin{array}{l}\text { LT de } 13,8 \mathrm{kv} \text { no trecho Dourados/Itaporã cruzando } \\
6 \mathrm{~km} \text { dentro da Al. }\end{array}$ \\
\hline Erikbaktsá & MT & 79.935 & 465 & $\begin{array}{l}\text { Canoeiros, } \\
\text { Erikbaktsá }\end{array}$ & $\begin{array}{l}\text { UHE J. Mirim planejada; LT de } 230 \text { kv planejada } \\
\text { atravessará a Al. }\end{array}$ \\
\hline Escondido & MT & 169.139 & 40 & Erikpatsa & $\begin{array}{l}\text { UHE Augusto: inventário da Bacia do Rio Tapajós } \\
\text { realizado em } 1988 .\end{array}$ \\
\hline Fazenda Guaraní & MG & 3.270 & 175 & Krenák, Pataxó & LT com extensão de $4 \mathrm{~km}$, tensão de $13,8 \mathrm{kv}$. \\
\hline Fulniô & PE & 11.506 & 2.790 & Fulniô & $\begin{array}{l}\text { LT com extensão de } 12 \mathrm{~km} \text { cruzando Al em } 288 \mathrm{ha} \text {. } \\
\text { Outra LT com extensão de } 25 \mathrm{~km} \text {. Impactos } \\
\text { ambientais identificados: desmatamento } \\
\text { e diminuição da caça. }\end{array}$ \\
\hline Funil & $\mathrm{TO}$ & 15.704 & 190 & Xerénte & $\begin{array}{l}\text { UHE Lajeado planejada. LT Celtins cruza Al com } \\
\text { extensão de } 12 \mathrm{~km} \text {, no trecho Funil/Tocantínia- } \\
\text { Miracema. LT trecho Tocantínia/Rio do Sono } \\
\text { com ampliação para } 34,5 \mathrm{kv} \text {. }\end{array}$ \\
\hline Gavião & AM & 8.612 & 45 & Múra & $\begin{array}{l}\text { UHE São Jirau Planejada; UHE Monte Cristo } \\
\text { planejada; UHE Santo Antônio planejada; } \\
\text { UHE Madeira Bin planejada. }\end{array}$ \\
\hline Guapenu & AM & 2.450 & 180 & Múra & $\begin{array}{l}\text { UHE São Jirau planejada; UHE Monte Cristo } \\
\text { planejada; UHE Santo Antônio planejada; } \\
\text { UHE Madeira Bin planejada. }\end{array}$ \\
\hline Guarita & RS & 23.406 & 3.929 & $\begin{array}{l}\text { Guaraní } \\
\text { e Kaingáng }\end{array}$ & $\begin{array}{l}\mathrm{LT} \text { cruzando a } \mathrm{Al} \text { em aproximadamente } 20 \mathrm{~km} \\
\text { de extensão. }\end{array}$ \\
\hline Ibirama & SC & 14.156 & 1.150 & $\begin{array}{l}\text { Guaraní, Kaingáng, } \\
\text { Xokléng }\end{array}$ & LT de $40 \mathrm{~km}$ de extensão. \\
\hline Ibotirama & BA & 2.020 & 448 & Tuxa & $\begin{array}{l}\text { UHE Luiz Gonzaga em operação e com } \\
\text { interferência direta. }\end{array}$ \\
\hline Içana Aiari & AM & 266.909 & 828 & $\begin{array}{l}\text { Baníwa, Korewa, } \\
\text { Kuripáka }\end{array}$ & UHE São Gabriel planejada. \\
\hline Igarapé Lage & $\mathrm{RO}$ & 107.321 & 233 & Pakaanóva & $\begin{array}{l}\text { UHE São Jirau planejada; UHE Monte Cristo } \\
\text { planejada; UHE Santo Antônio planejada; } \\
\text { UHE Madeira Bin planejada. }\end{array}$ \\
\hline Igarapé Lourdes & $\mathrm{RO}$ & 185.534 & 446 & Gavião & $\begin{array}{l}\text { Projeto básico da UHE Ji-Paraná se efetivado } \\
\text { inundará } 11.000 \text { ha. }\end{array}$ \\
\hline Igarapé Ribeirão & $\mathrm{RO}$ & 47.863 & 102 & Pakaanóva & $\begin{array}{l}\text { UHE São Jirau, UHE Monte Cristo, UHE } \\
\text { Santo Antônio e UHE Madeira Bin planejadas. }\end{array}$ \\
\hline Inhancora & RS & 2.841 & 444 & Kaingáng & LT com extensão de $800 \mathrm{~m}$ dentro da área. \\
\hline Ipixuna & AM & 179.640 & 211 & Paritintín & $\begin{array}{l}\text { UHE São Jirau, UHE Monte Cristo, UHE } \\
\text { Santo Antônio e UHE Madeira Bin planejadas. }\end{array}$ \\
\hline Itaitinga & AM & 160 & 25 & Múra & $\begin{array}{l}\text { UHE São Jirau, UHE Monte Cristo, UHE } \\
\text { Santo Antônio e UHE Madeira Bin planejadas. }\end{array}$ \\
\hline Japuíra & MT & 152.509 & 98 & Erikbaktsá, Canoeiro & UHE J. Mirim planejada. \\
\hline Jarára & MS & 479 & 250 & Guaraní-Kayowa & $\begin{array}{l}\text { UHE Ilha Grande planejada, projeto aprovado } \\
\text { pelo DNAEE. }\end{array}$ \\
\hline
\end{tabular}

(continua) 
Tabela 1 (continuação)

\begin{tabular}{|c|c|c|c|c|c|}
\hline Área indígena & Estado & Superficie (km²) & População & Grupo indígena & Tipo de interferência \\
\hline Jumas & AM & a definir & 46 & Múra & $\begin{array}{l}\text { UHE São Jirau, UHE Monte Cristo, UHE } \\
\text { Santo Antônio e UHE Madeira Bin planejadas. }\end{array}$ \\
\hline Kaingáng de Irai & RS & 235 & 294 & Kaingáng & $\begin{array}{l}\text { UHE Itapiranga planejada; LT com uma extensão } \\
\text { de } 0,25 \mathrm{~km} \text { construída em } 1988 \text {. Fornece energia } \\
\text { elétrica para os índios de forma precária. }\end{array}$ \\
\hline Kararaô & PA & 224.000 & 27 & Kararaô & UHE Complexo do Xingu planejado. \\
\hline Karipúna & RO & 195.000 & 13 & Karipúna & $\begin{array}{l}\text { UHE São Jirau, UHE Monte Cristo, UHE } \\
\text { Santo Antônio e UHE Madeira Bin planejadas. }\end{array}$ \\
\hline Karirí-Xoco & $\mathrm{AL}$ & 699 & 1.500 & Karirí-Xoco & $\begin{array}{l}\text { UHE Orocó planejada; LT para abastecimento } \\
\text { de São Braz, com extensão de } 1 \mathrm{~km} \text {; LT para } \\
\text { abastecimento de Porto Real do Colégio } \\
\text { com extensão de } 1 \mathrm{~km} \text {. }\end{array}$ \\
\hline Karitiána & $\mathrm{RO}$ & 89.682 & 175 & Karipúna & $\begin{array}{l}\text { UHE Samuel em operação; UHE São Jirau, } \\
\text { UHE Monte Cristo, UHE Santo Antônio } \\
\text { e UHE Madeira Bin planejadas. }\end{array}$ \\
\hline Kayabí & PA & 117.247 & 401 & Kayabí & $\begin{array}{l}\text { UHE Apiacas com construção paralisada; UHE Sete } \\
\text { Quedas planejada; UHE Celita planejada; Inventário } \\
\text { da Bacia do Rio Tapajós realizado em } 1988 .\end{array}$ \\
\hline Coatinema & PA & 388.304 & 68 & Asuriní & UHE Complexo do Xingu planejada. \\
\hline Kraolândia & TO & 302.533 & 1.198 & Krahô & $\begin{array}{l}\text { UHE Santo Antônio do Tocantins e UHE Lajeado } \\
\text { planejadas. }\end{array}$ \\
\hline Krenák & MG & 1.791 & 112 & Krenák & LT de $110 \mathrm{kv}$. \\
\hline Krikatí & MA & 146.000 & 470 & Krikatí, Guajajára & $\begin{array}{l}\text { UHE Tucuruí em operação; UHE Santo Antônio } \\
\text { do Tocantins. }\end{array}$ \\
\hline Kuripáka & AM & 116.443 & 324 & Kobewa & UHE São Gabriel planejada. \\
\hline Ligeiro & RS & 4.565 & 920 & Kaingáng & $\begin{array}{l}\text { UHE Machadinho. Alagamento de terras de alta } \\
\text { qualidade com perda } 188 \mathrm{ha} \text { ( } 5 \% \text { da Al); LT de } 89 \mathrm{kv} \\
\text { cruzando área de } 7 \mathrm{~km} \text { de extensão e } 150 \mathrm{~m} \text { de largura. }\end{array}$ \\
\hline Mãe Maria & PA & 62.488 & 281 & Gavião & $\begin{array}{l}\text { UHE Marabá planejada; LT trecho Marabá/Imperatriz: } \\
\text { faixa paralela a BR-322 de } 25 \mathrm{~km} \text { de extensão. }\end{array}$ \\
\hline Manguerinha & PR & 16.375 & 1.280 & Kaingáng, Guaraní & $\begin{array}{l}\text { UHE Salto Santiago com alagamento de 30,5ha; } 2 \text { LT } \\
\text { de 230kv; UHE Fundão, UHE Jacú e UHE Pinhão } \\
\text { planejadas; UHE Segredo em construção; LT Morro } \\
\text { Verde/Capinzinho. }\end{array}$ \\
\hline Marrecas & PR & 16.839 & 517 & Kaingáng & UHE Taguá planejada. \\
\hline Masaká & $\mathrm{RO}$ & 315.000 & 0 & Isolados & $\begin{array}{l}\text { UHE São Jirau, UHE Monte Cristo, UHE } \\
\text { Santo Antônio e UHE Madeira Bin planejadas. }\end{array}$ \\
\hline $\begin{array}{l}\text { Maxacalí Aldeia } \\
\text { Velho Pradinho }\end{array}$ & MG & 1.028 & 309 & Maxacalí & $\begin{array}{l}\text { LT com extensão de } 3 \mathrm{~km} \text { corta a Al. Impactos } \\
\text { ambientais identificados: desmatamento e trânsito } \\
\text { não autorizado de pessoas. }\end{array}$ \\
\hline Meria & AM & 663 & 75 & Miranha & UHE São Gabriel planejada. \\
\hline Merure & MT & 83.301 & 473 & Boróro & $\begin{array}{l}\text { UHE Torixoréu planejada; UHE Barra do Peixe: } \\
\text { estudo de viabilidade; UHE Couto Magalhães: } \\
\text { estudo de viabilidade; UHE Foz do Nodoire: estudo } \\
\text { de viabilidade; LT planejada cortará a Al. }\end{array}$ \\
\hline Mundurukú & PA & 948.541 & 3.269 & Mundurukú & $\begin{array}{l}\text { UHE Itaituba planejada; Inventário da Bacia } \\
\text { do Rio Tapajós realizado em } 1988 .\end{array}$ \\
\hline Murutinga & AM & 1.270 & 85 & Múra & $\begin{array}{l}\text { UHE São Jirau, UHE Monte Cristo, UHE } \\
\text { Santo Antônio e UHE Madeira Bin planejadas. }\end{array}$ \\
\hline Nambikwára & MT & 1.011 .961 & 160 & Nambikwára & $\begin{array}{l}\text { UHE } 12 \text { de Outubro: estudo de viabilidade. } \\
\text { Interferência direta com alagamento de } 4,4 \text { ha na Al. }\end{array}$ \\
\hline
\end{tabular}

(continua) 
Tabela 1 (continuação)

\begin{tabular}{|c|c|c|c|c|c|}
\hline Área indígena & Estado & Superficie $\left(\mathrm{km}^{2}\right)$ & População & Grupo indígena & Tipo de interferência \\
\hline Natal Felicidade & AM & 313 & 60 & Múra & $\begin{array}{l}\text { UHE São Jirau, UHE Monte Cristo, UHE } \\
\text { Santo Antônio e UHE Madeira Bin planejadas. }\end{array}$ \\
\hline Nhamunda Mapuera & AM & 1.049 .520 & 464 & $\begin{array}{l}\text { Hixkaryána, } \\
\text { Waiwái }\end{array}$ & $\begin{array}{l}\text { UHE Cachoeira Porteira: acordo com concessionária } \\
\text { não formalizado. Se construída poderá atingir a Al } \\
\text { e área de perambulação. }\end{array}$ \\
\hline Nonoai & RS & 14.910 & 2.480 & $\begin{array}{l}\text { Kaingáng } \\
\text { e Guaraní }\end{array}$ & $\begin{array}{l}\text { LT com extensão de } 1 \mathrm{~km} \text {, sem compensação. Linhas } \\
\text { de distribuição solicitadas pela FUNAl e comunidade. }\end{array}$ \\
\hline Pankararu & AM & 234.400 & 290 & Paritintín & $\begin{array}{l}\text { UHE Luiz Gonzaga em operação. Impacto: } \\
\text { diminuição da caça, aumento da distância a } \\
\text { Petrolândia, circulação de posseiros pela área } \\
\text { e inundação de espaço sagrado; LT de } 9 \mathrm{~km} \\
\text { no trecho Paulo Afonso/Fortaleza: solo eletrificado, } \\
\text { desmatamento e redução da caça e área agrícola. }\end{array}$ \\
\hline Ofayé Xavánte & MS & 1.937 & 44 & Ofayé-Xavánte & $\begin{array}{l}\text { UHE Porto Primavera/CESP: construção adiada para } \\
\text { 1984; Impacto direto: não foi dada solução para } \\
\text { a relocação dos índios (terra indígena ainda não foi } \\
\text { identificada). FUNAI promoveu reuniões para } \\
\text { solucionar a questão (1990). }\end{array}$ \\
\hline Onça & AM & 413 & 0 & Múra & $\begin{array}{l}\text { UHE São Jirau planejada; UHE Monte Cristo } \\
\text { planejada; UHE Santo Antônio planejada; } \\
\text { UHE Madeira Bin planejada. }\end{array}$ \\
\hline Pacaás Novas & $\mathrm{RO}$ & 279.906 & 438 & Pakaanóva & $\begin{array}{l}\text { UHE São Jirau planejada; UHE Monte Cristo } \\
\text { planejada; UHE Santo Antônio planejada; } \\
\text { UHE Madeira Bin planejada. }\end{array}$ \\
\hline Padre & AM & 840 & 45 & Múra & $\begin{array}{l}\text { UHE São Jirau planejada; UHE Monte Cristo } \\
\text { planejada; UHE Santo Antônio planejada; } \\
\text { UHE Madeira Bin planejada. }\end{array}$ \\
\hline Palmas PR & $\mathrm{SC}$ & 2.944 & 770 & Kaingáng & $\begin{array}{l}\text { LT/COPEL: extensão de } 2 \mathrm{~km} \text {, com rede de } \\
\text { abastecimento e sem compensação. }\end{array}$ \\
\hline Panambi & MS & 2.037 & 470 & Guaraní-Kaiwá & $\begin{array}{l}\text { LT Douradinha/Piraporã/ENERSUL de } 34,8 \mathrm{kv} \text {, } \\
\text { com extensão de } 200 \mathrm{~m} \text { (1991) margeia a Al } \\
\text { com ramificação. Houve compensação. }\end{array}$ \\
\hline Pankarare & BA & 29.597 & 1.039 & Pankarare & $\begin{array}{l}\text { UHE Moxoxó/CHESF em operação; UHE Paulo } \\
\text { Afonso/CHESF em operação; UHE Luiz Gonzaga/ } \\
\text { CHESF em operação. }\end{array}$ \\
\hline Pankararu & PE & 8.337 & 4.087 & Pankararu & $\begin{array}{l}\text { UHE Luiz Gonzaga/CHESF em operação. Impacto: } \\
\text { redução da caça, aumento da distância a } \\
\text { Petrolândia, maior número de posseiros na área, } \\
\text { acampamento e vila residencial vizinhos a Al } \\
\text { e inundação de espaço sagrado; LT/CHESF: extensão } \\
\text { de } 9 \text { km no trecho Paulo Afonso/Fortaleza, linha } \\
\text { distante } 20 \mathrm{~km} \text { do acampamento Itaparica. Impactos: } \\
\text { solo eletrificado e diminuição da agricultura } \\
\text { e da caça. UHE Complexo do Xingu planejada. }\end{array}$ \\
\hline Paquiçamba & PA & 4.355 & 35 & Jurúna & UHE Complexo do Xingu planejada. \\
\hline Paracuhuba & AM & 927 & 35 & Múra & $\begin{array}{l}\text { UHE São Jirau, UHE Monte Cristo, UHE } \\
\text { Santo Antônio e UHE Madeira Bin planejadas. }\end{array}$ \\
\hline Parakanã & PA & 351.697 & 231 & Parakanã & $\begin{array}{l}\text { UHE Tucuruí em operação. Impactos: proliferação } \\
\text { de mosquitos subsequente ao enchimento do } \\
\text { reservatório, perdas territoriais por alagamento, } \\
\text { dermatoses e disseminação de agentes infecciosos. }\end{array}$ \\
\hline Pari Cahoeira I & AM & 353.027 & 1.600 & Makú, Tukáno & UHE São Gabriel planejada. \\
\hline Pari Cachoeira II & AM & 155.336 & 2.082 & Tukáno, Makú & UHE São Gabriel planejada. \\
\hline Pari Cachoeira III & AM & 11.158 & 1.200 & Makú, Tukáno & UHE São Gabriel planejada. \\
\hline
\end{tabular}

(continua) 
Tabela 1 (continuação)

\begin{tabular}{|c|c|c|c|c|c|}
\hline Área indígena & Estado & Superficie $\left(\mathrm{km}^{2}\right)$ & População & Grupo indígena & Tipo de interferência \\
\hline Paritintín & $\mathrm{RO}$ & a definir & 0 & Paritintín & $\begin{array}{l}\text { UHE São Jirau, UHE Monte Cristo, UHE } \\
\text { Santo Antônio e UHE Madeira Bin planejadas. }\end{array}$ \\
\hline Parque do Araguaia & $\mathrm{TO}$ & 1.395 .000 & 1.048 & $\begin{array}{l}\text { Avá-Canoeiro, } \\
\text { Javaé, Karajá, } \\
\text { Tapirapé }\end{array}$ & $\begin{array}{l}\text { LT segue paralela a estrada que dá acesso } \\
\text { a Formoso do Araguaia e Gurupi. Encontra-se } \\
\text { defronte a aldeia, do outro lado do Rio Javaé. }\end{array}$ \\
\hline Pimentel Barbosa & $\mathrm{MT}$ & 328.966 & 375 & Xavánte & $\begin{array}{l}\text { UHE Torixoréu planejada; UHE Barra do Peixe: } \\
\text { estudo de viabilidade com impacto indireto; UHE } \\
\text { Couto Magalhães: estudo de viabilidade; UHE Foz } \\
\text { do Nodoire: estudo de viabilidade paralisado. }\end{array}$ \\
\hline Pirahã & AM & 389.000 & 179 & Pirahã & $\begin{array}{l}\text { UHE São Jirau, UHE Monte Cristo, UHE } \\
\text { Santo Antônio e UHE Madeira Bin planejadas. }\end{array}$ \\
\hline Pirajuí & MS & 2.118 & 1.497 & Guaraní Nhandéva & $\begin{array}{l}\text { UHE Ilha Grande planejada; LT Sete Quedas/ } \\
\text { Paranhos de } 34,5 \mathrm{kv}: 3 \mathrm{~km} \text { dentro da Al. }\end{array}$ \\
\hline Pinineus de Souza & MT & 28.212 & 135 & Nambikwára & $\begin{array}{l}\text { UHE } 12 \text { de Outubro: estudo de viabilidade. } \\
\text { Interferência direta: usina a fio d'água inunda } 4,4 \text { ha, } \\
\text { com comprometimento das áreas de caça, pesca } \\
\text { e seringal. }\end{array}$ \\
\hline Piripicura & MT & a definir & 0 & Kawahibe & UHE Matamaté planejada. \\
\hline Porto Lindo & MS & 1.650 & 1.600 & Guaraní Nhandéva & UHE Ilha Grande planejada. \\
\hline Potiguara & PB & 21.238 & 6.156 & Potiguara & $\begin{array}{l}\text { LT trecho Rio Tinto/Baía da Traição. Introdução } \\
\text { de novos hábitos. }\end{array}$ \\
\hline Praia do Índio & PA & 28 & 4 & Mundurukú & UHE Itaituba planejada. \\
\hline Praia do Mangue & PA & 30 & 0 & Mundurukú & UHE Itaituba planejada. \\
\hline Queimadas & PR & 3.081 & 327 & Kaingáng & $\begin{array}{l}\text { UHE Mauá: inventário; LT corta área em uma faixa } \\
\text { de } 150 \mathrm{~m} \text { de largura e } 5 \mathrm{~km} \text { de extensão; LT Linhão } \\
\text { de Itaipu com } 4 \mathrm{~km} \text { de extensão no trecho Ivaiporã/ } \\
\text { São Paulo. Interferência direta: desmatamento } \\
\text { e diminuição da áreas de coleta e extrativismo. }\end{array}$ \\
\hline Recreio São Félix & AM & 251 & 130 & Múra & $\begin{array}{l}\text { UHE São Jirau, UHE Monte Cristo, UHE } \\
\text { Santo Antônio e UHE Madeira Bin planejadas. }\end{array}$ \\
\hline Rio Branco & $\mathrm{RO}$ & 236.137 & 306 & Makuráp, Tuparí & $\begin{array}{l}\text { PCH Cachimbo planejada; UHE Ji-Paraná; } \\
\text { PCH Alta Floresta. }\end{array}$ \\
\hline Rio da Várzea & RS & 16.100 & 265 & Kaingáng & $\begin{array}{l}\text { LT atravessando a } \mathrm{Al} \text { em } 3 \mathrm{~km} \text {. Todos os moradores } \\
\text { da Al possuem luz nas moradias. }\end{array}$ \\
\hline Rio Formoso & MT & 19.749 & 79 & Pareci & $\begin{array}{l}\text { Concessão à Itamarati S/A Agropecuária para } \\
\text { construção de duas usinas com } 42 \mathrm{kv} \text { de } \\
\text { aproveitamento hidráulico para produção de energia } \\
\text { elétrica no Rio Juba distando } 10 \mathrm{~km} \text { do limite sul da } \\
\text { Al. Provável impacto direto. }\end{array}$ \\
\hline Rio Guaporé & $\mathrm{RO}$ & 115.796 & 227 & Jabutí, Makuráp & $\begin{array}{l}\text { UHE São Jirau, UHE Monte Cristo, UHE } \\
\text { Santo Antônio e UHE Madeira Bin planejadas. }\end{array}$ \\
\hline Rio Paru de Este & PA & 1.182 .800 & 68 & Apalaí, Wanána & UHE Paru Novo planejada. \\
\hline Rodelas & $\mathrm{BA}$ & 104 & 486 & Tuxa & $\begin{array}{l}\text { LT/COELBA; Barragem de Itaparica/CHESF inundou } \\
\text { toda a área do PI Rodelas. Os índios tiveram que ser } \\
\text { removidos para outra área. Negociação entre } \\
\text { CHESF/FUNAI ainda não está concluída. }\end{array}$ \\
\hline Sagarana & $\mathrm{RO}$ & 17.800 & 148 & Pakaanóva & $\begin{array}{l}\text { UHE São Jirau, UHE Monte Cristo, UHE } \\
\text { Santo Antônio e UHE Madeira Bin planejadas. }\end{array}$ \\
\hline $\begin{array}{l}\text { Sangradouro Volta } \\
\text { Grande }\end{array}$ & $\mathrm{MT}$ & 100.280 & 755 & Xavánte, Boróro & $\begin{array}{l}\text { UHE Torixoréu planejada; UHE Barra do Peixe: } \\
\text { estudo de viabilidade/ELETRONORTE, impacto } \\
\text { indireto; UHE Couto Magalhães: estudo de } \\
\text { viabilidade/ELETRONORTE; UHE Foz do Nodoire: } \\
\text { estudo de viabilidade paralisado/CEMAT (1989). }\end{array}$ \\
\hline
\end{tabular}

(continua) 
Tabela 1 (continuação)

\begin{tabular}{|c|c|c|c|c|c|}
\hline Área indígena & Estado & Superficie $\left(\mathrm{km}^{2}\right)$ & População & Grupo indígena & Tipo de interferência \\
\hline Santana & MT & 35.471 & 192 & Bakairí & LT/CEMAT de 34,5kv planejada atravessará a área. \\
\hline São Domingos & MT & 5.705 & 109 & Karajá & LT/CEMAT de $138 \mathrm{kv}$ planejada atravessará a área. \\
\hline São Marcos & MT & 188.478 & 1.934 & Xavánte & $\begin{array}{l}\text { UHE Torixoréu planejada; UHE Barra do Peixe: } \\
\text { estudo de viabilidade/ELETRONORTE (1988), } \\
\text { impacto indireto; UHE Couto Magalhães: estudo de } \\
\text { viabilidade/ELETRONORTE; UHE Foz do Nodoire: } \\
\text { estudo de viabilidade paralisado/CEMAT (1989); } \\
\text { LT/CEMAT de 34,5kv fará limite com Al. }\end{array}$ \\
\hline São Pedro & AM & 726 & 20 & Múra & $\begin{array}{l}\text { UHE São Jirau, UHE Monte Cristo, UHE } \\
\text { Santo Antônio e UHE Madeira Bin planejadas. }\end{array}$ \\
\hline Sapucaia & AM & 106.150 & 0 & Múra & $\begin{array}{l}\text { UHE São Jirau, UHE Monte Cristo, UHE } \\
\text { Santo Antônio e UHE Madeira Bin planejadas. }\end{array}$ \\
\hline Sarare & MT & 67.420 & 66 & Manairisu & Duas LT planejadas com 138kv atravessarão a área. \\
\hline Sassoro & MS & 1.923 & 1.477 & Guaraní Kaiwá & UHE Ilha Grande planejada. \\
\hline Serra Morena & MT & 147.836 & 177 & Cinta-Larga & $\begin{array}{l}\text { PCH Juína: construção paralisada com barragem } \\
\text { e casa de máquinas prontas. Impacto indireto: } \\
\text { previsão de inundação de } 193 \mathrm{ha} \text {. }\end{array}$ \\
\hline Sete Setembro MT & $\mathrm{RO}$ & 248.147 & 551 & Suruí & UHE Ji-Paraná (projeto básico). \\
\hline Sororo & PA & 26.257 & 142 & Aikemar & UHE Santa Isabel planejada. \\
\hline Tadarimana & MT & 9.785 & 177 & Boróro & $\begin{array}{l}\text { LT de } 138 \mathrm{kv} \text { atravessando a área em } 6 \mathrm{~km} \text {; LT } \\
\text { de } 230 \mathrm{kv} \text { tangenciando a área. }\end{array}$ \\
\hline Takuaraty Yvykuarusu & MS & 2.475 & 360 & Guaraní Kaiwá & UHE Ilha Grande planejada. \\
\hline Tapiraré/Karajá & MT & 66.166 & 394 & Tapiraré/Karajá & LT de $138 \mathrm{kv}$ planejada atravessará área. \\
\hline Taquaperi & MS & 1.886 & 1.692 & Guaraní Kaiwá & UHE Ilha Grande planejada. \\
\hline Taracua & AM & 480.414 & 1.400 & $\begin{array}{l}\text { Arapáso, Desáno, } \\
\text { Tukáno e outros }\end{array}$ & UHE São Gabriel planejada. \\
\hline Tenharim Igarapé Preto & AM & 79.500 & 42 & Tenharim & UHE Matamaté planejada. \\
\hline Tenharim Marmelos & AM & 488.550 & 275 & Tenharim & $\begin{array}{l}\text { UHE São Jirau, UHE Monte Cristo, UHE } \\
\text { Santo Antônio e UHE Madeira Bin planejadas. }\end{array}$ \\
\hline Teresa Cristina & MT & 35.864 & 285 & Boróro & $\begin{array}{l}\text { LT de } 138 \mathrm{kv} \text { corta a área em aproximadamente } \\
30 \mathrm{~km} \text {. }\end{array}$ \\
\hline Tibagy & PR & 859 & 78 & Kaingáng & UHE São Jerônimo planejada. \\
\hline Tingui Boto & $\mathrm{AL}$ & 122 & 154 & Tingui-Boto & UHE Orocó e UHE Ibó planejadas. \\
\hline Toldo Chimbangue & SC & 988 & 240 & Kaingáng & $\begin{array}{l}\text { UHE Itá/ELETROSUL; LT com extensão de } 5 \mathrm{~km} \\
\text { com fornecimento de energia para toda a área. }\end{array}$ \\
\hline Torá & AM & 24.600 & 66 & Paritintín & $\begin{array}{l}\text { UHE São Jirau, UHE Monte Cristo, UHE } \\
\text { Santo Antônio e UHE Madeira Bin planejadas. }\end{array}$ \\
\hline Tracajá & AM & 690 & 20 & Múra & $\begin{array}{l}\text { UHE São Jirau, UHE Monte Cristo, UHE } \\
\text { Santo Antônio e UHE Madeira Bin planejadas. }\end{array}$ \\
\hline Trincheira & AM & 1.623 & 200 & Múra & $\begin{array}{l}\text { UHE São Jirau, UHE Monte Cristo, UHE } \\
\text { Santo Antônio e UHE Madeira Bin planejadas. }\end{array}$ \\
\hline Trocara & PA & 21.723 & 160 & Asuriní & $\begin{array}{l}\text { UHE Tucuruí em operação. Interferência indireta: } \\
\text { barragem encontra-se a } 18 \mathrm{~km} \text { da Al. }\end{array}$ \\
\hline Truka & PE & 1.650 & 909 & Truka & $\begin{array}{l}\text { LT com extensão de } 13 \mathrm{~km} \text {. Impactos: introdução } \\
\text { de novos hábitos e tecnologia. }\end{array}$ \\
\hline Tubarão Latundê & RO & 116.613 & 160 & Aikaná, Latundê & $\begin{array}{l}\text { UHE Ávila planejada. Interferência direta: atinge } \\
\text { parte do limite leste da Al com } 4.000 \text { ha inundados. }\end{array}$ \\
\hline Tumucumaque & PA & 2.700 .000 & 1.033 & Apalaí, Wayána & UHE Paru Novo planejada. \\
\hline
\end{tabular}

(continua) 


\begin{tabular}{|c|c|c|c|c|c|}
\hline Área indígena & Estado & Superficie $\left(\mathbf{k m}^{2}\right)$ & População & Grupo indígena & Tipo de interferência \\
\hline Umutina & MT & 28.120 & 252 & Barbados, Pareci & $\begin{array}{l}\text { LT de } 34,5 \mathrm{kv} \text { corta a área; UHE Juba I e II: } \\
\text { impacto direto. }\end{array}$ \\
\hline Uneiuxi & AM & 405.000 & 340 & Makú, Tukáno & UHE São Gabriel planejada. \\
\hline Vale do Guaporé & MT & 242.593 & 344 & $\begin{array}{l}\text { Nambikwára, } \\
\text { Manairisu }\end{array}$ & LT de 138kv planejada atravessará a área. \\
\hline Vanuire & SP & 708 & 189 & Kaingáng & $\begin{array}{l}\text { LT Tupã/B. Ponte Alta abrangendo } 2,5 \mathrm{~km} \text { dentro } \\
\text { área. Luz elétrica nas residências indígenas. }\end{array}$ \\
\hline Votouro & RS & 1.865 & 797 & $\begin{array}{l}\text { Guaraní } \\
\text { e Kaingáng }\end{array}$ & $\begin{array}{l}\text { LT Entre-Rios/Eval Grande com uma extensão } \\
\text { de } 2 \mathrm{~km} \text { dentro da área. }\end{array}$ \\
\hline Waiãpi & AP & 573.000 & 366 & Waiãpi & UHE Coaracy Nunes em operação. \\
\hline Waimirí Atroarí AM & $\mathrm{RR}$ & 2.585 .911 & 380 & Waimirí Atroarí & $\begin{array}{l}\text { UHE Balbina em operação. Interferência direta. } \\
\text { Programa Waimirí-Atroarí (1992). }\end{array}$ \\
\hline Xambioá & TO & 3.265 & 193 & Karajá, Guaraní & UHE Santa Isabel e UHE Serra Quebrada planejadas. \\
\hline Xapecó & SC & 15.623 & 3.560 & Kaingáng, Guaraní & $\begin{array}{l}\text { UHE Voltão Novo planejada; UHE Xanxerê } \\
\text { (alagamento de 200ha). UHE Salto Manela (obras } \\
\text { iniciadas e paralisadas com alagamento de 180ha); } \\
\text { LT de } 23 \mathrm{kv} \text { corta Al em } 7.031 \mathrm{~m} \text {. }\end{array}$ \\
\hline Xerénte & TO & 167.542 & 1.371 & Xerénte & UHE Lajeado e UHE Sono (I, II, III) planejadas. \\
\hline Xingu Bacajá & PA & 192.127 & 218 & Xikrín & UHE Complexo do Xingu planejado. \\
\hline Xipáya Curua & PA & 13.000 & 51 & Xipáya, Curuya & UHE Complexo do Xingu planejado. \\
\hline Yanomámi RR & AM & 9.419 .108 & 10.960 & Yanomámi & $\begin{array}{l}\text { UHE São Gabriel planejada; UHE Paredão (estudo } \\
\text { de viabilidade, interferência direta). }\end{array}$ \\
\hline Yawareté I & AM & 347.326 & 3.276 & $\begin{array}{l}\text { Makú, Kobewa, } \\
\text { Tukáno }\end{array}$ & UHE São Gabriel planejada. \\
\hline Apiaká Kayabí & MS & 109.245 & 285 & Apiaká Kayabí & $\begin{array}{l}\text { Tentativa de liberação do Salto Kayabí para } \\
\text { construção de UHE em troca de terras já ocupadas } \\
\text { por população externa. Visita de técnicos ao local } \\
\text { sem consentimento. }\end{array}$ \\
\hline Kayapó & PA & 3.284 .005 & 1.823 & Kayapó & UHE Kokraimôro e UHE Jarina planejadas. \\
\hline Barão de Antonina & PR & 3.751 & 359 & Kaingáng & $\begin{array}{l}\text { UHE Cebolão planejada. Duas LT cruzando Al, } \\
\text { ambas com } 14 \mathrm{~km} \text { de extensão. }\end{array}$ \\
\hline
\end{tabular}

Al: Área Indígena; CEMAT: Centrais Elétricas Matogrossenses; CESP: Companhia Energética de São Paulo; CHESF: Companhia Hidro Elétrica do São Francisco; COELBA: Companhia Elétrica do Estado da Bahia; COPEL: Companhia Paranaense de Energia; DNAEE: Departamento Nacional de Águas e Energia Elétrica; ELETRONORTE: Centrais Elétricas do Norte do Brasil; ENERSUL: Empresa Energética do Estado do Mato Grosso do Sul; FUNAI: Fundação Nacional do Índio; FURNAS: Furnas Centrais Elétricas; LT: Linha de Transmissão; PCH: Pequena Central Hidrelétrica; PI: Parque Indígena; UHE: Usina Hidrelétrica. Fonte: Fundação Nacional do Índio, Ministério da Justiça.

Em relação às usinas hidrelétricas planejadas, alguns exemplos tipificam as dimensões do problema em análise. Assim, a construção das usinas hidrelétricas Monte Cristo, Santo Antonio, São Jirau e Madeira Bin afetaria 32 áreas indígenas nos estados do Amazonas e Rondônia (comunidades Múra, Sateré-Mawé, Parintintín, Pirahã, Tenharím, Pakaanóva, Karipúna e Munduama), totalizando 5.123 pessoas, para não mencionar os grupos ainda isolados vivendo na região.

Outro exemplo é o da construção, em Mato Grosso, das usinas hidrelétricas de Torixoréu (planejada) e aquelas em estudo de viabilidade (Barra do Peixe, Couto Magalhães e da Foz do Nodoire), cuja entrada em funcionamento afetará diretamente ao redor de 4.000 indígenas das nações Xavánte e Boróro. Ainda neste sentido, a construção do complexo Altamira no Rio Xingu, no Pará, inundará cerca de $7.400 \mathrm{~km}^{2}$, atingindo as áreas indígenas Kararaô, Mekragorotire, Gorotire, Kikretum, Kubenkrankégn, Aukre, Kokraimôro, Xikrín, Araweté, Asuriní, Arára, Parakanã, Jurúna, Curukua e Xipáya, totalizando cerca de 3.600 pessoas (Mullen, 1991). 
Uma das experiências de implantação de barragens pelo setor elétrico mais estudadas é a da hidrelétrica de Tucuruí no Pará, a qual inundou $2.430 \mathrm{~km}^{2}$, atingindo diretamente, através do alagamento, as áreas indígenas Asuriní, Gavião, Suruí, Parakanã e Xikrín, bem como as áreas Guajará e Krikatí pela construção das linhas de transmissão (Santos \& Nacke, 1988, 1991). Além dos efeitos diretos acarretados pelo alagamento de extensas áreas, como, por exemplo, o aumento da densidade de mosquitos (Bulcão, 1994; Couto, 1996; Figueiredo \& Ricci, 1989; Tadei, 1994) e a circulação do plasmódio em animais (Arruda, 1985), a utilização de potentes desfolhantes como o Tordon 101 BR (picloran 2,4 D) e Tordon 155 (picloran 2,4,5 T) para a limpeza das áreas de servidão das linhas de transmissão $(800 \mathrm{~km}$ de extensão e $100 \mathrm{~m}$ de largura) entre Tucuruí e Barcarena com fins de controlar o crescimento da vegetação, resultou na morte de animais, contaminação dos cursos de água e episódios de intoxicação exógena aguda (Couto, 1996). Em uma das comunidades indígenas afetadas na área de construção de Tucuruí, a dos Gaviões, observou-se na década seguinte a ocorrência inusitada de dois casos de câncer (uma leucemia e um sarcoma de partes moles) em adultos jovens da Área Indígena Mãe-Maria, vivendo nas proximidades de linhas de transmissão de alta tensão elétrica (Koifman et al., 1998; Vieira Filho, 1994). A observação destes casos de câncer adquire relevância, considerando-se o persistente debate na comunidade científica acerca da possível natureza causal da associação entre a exposição aos campos eletromagnéticos de baixa freqüên-

Figura 1

Hidrelétricas na Amazônia. Localização geográfica dos empreendimentos.

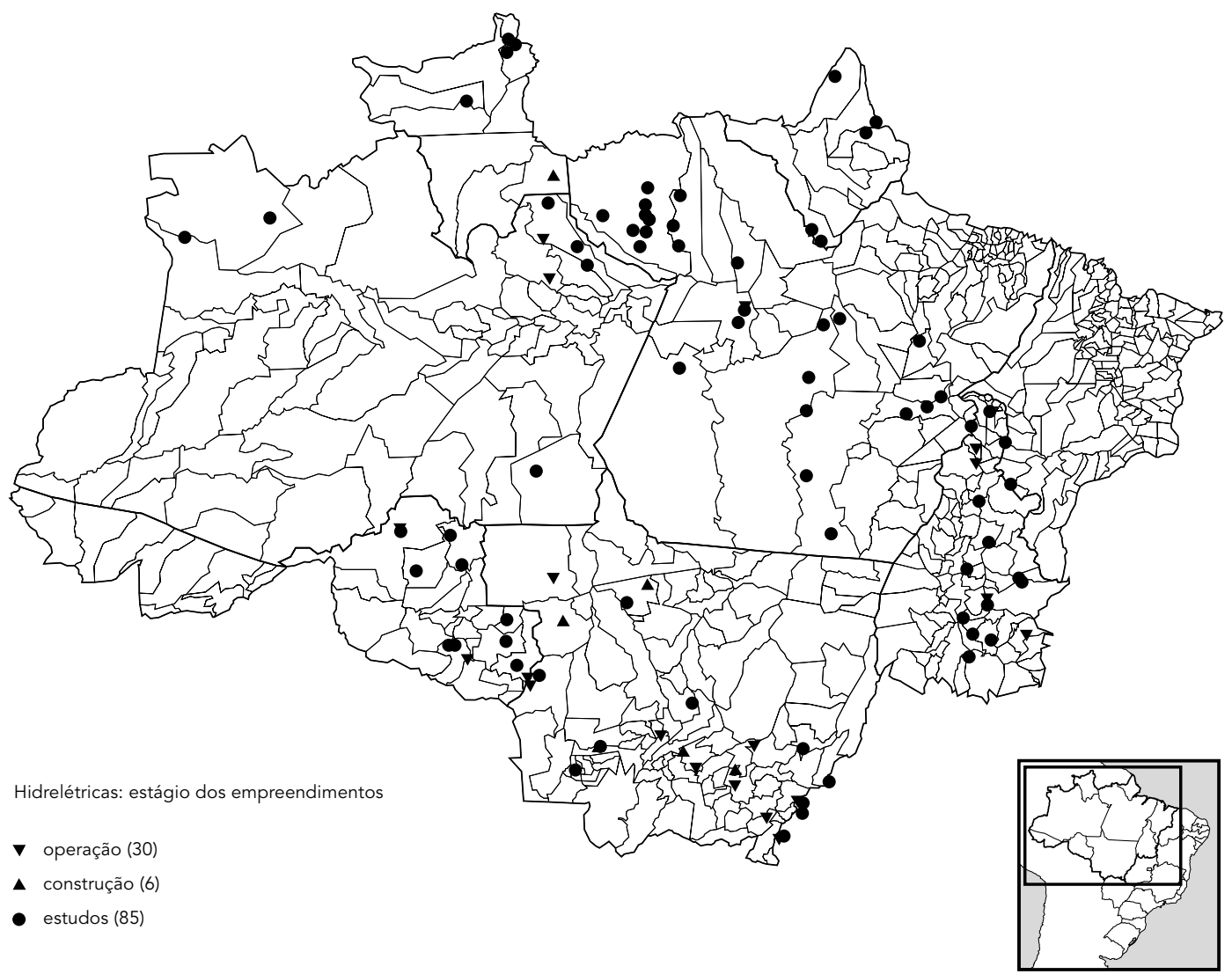

Obs: as hidrelétricas que não constam no mapa não estão georreferenciadas.

Fonte: ELETROBRÁS, Plano 2.015 (apud Couto, 1996). 
cia, como aquela observada nas proximidades de linhas de transmissão elétrica, e o desenvolvimento de tumores malignos, sobretudo leucemia e tumor de cérebro (Preece et al., 2000).

Sendo a região amazônica depositária das maiores reservas hídricas disponíveis com potencial hidrelétrico no país, a proposta de sua plena utilização deu lugar à elaboração do Plano 2.015 (Figura 1), que prevê a implantação de 121 usinas hidrelétricas naquela região, das quais 85 já estão em fase de estudos (Couto, 1996). Sucede que as áreas habitadas por diversas comunidades indígenas superpõem-se àquelas em processo de implantação de barragens, ou que serão direta ou indiretamente afetadas pela expansão desenhada para o setor elétrico. Assim, antecipa-se a ocorrência de amplo impacto sócio-econômico e epidemiológico sobre os povos indígenas da Amazônia.

Desta maneira, em futuro imediato, estaremos confrontando, por um lado, os benefícios

\section{Referências}

ARRUDA, M. E., 1985. Presença de plasmódio brasilianum em macacos capturados na área de enchimento do reservatório da usina hidroelétrica de Tucuruí, Pará. Memórias do Instituto Oswaldo Cruz, 80:367-369.

BULCÃO, J. A. P., 1994. Proposta de um Modelo para Avaliação do Impacto dos Empreendimentos Hidroelétricos sobre as Doenças Transmitidas por Vetores com Especial Referência à Malária. Dissertação de Mestrado, Rio de Janeiro: Instituto Oswaldo Cruz, Fundação Oswaldo Cruz.

CONSOLINI, J.; LUZ, E. \& TORRES, P. B., 1990. Flebótomos da área do reservatório da Hidroelétrica de Itaipu, Estado do Paraná, Brasil (Diptera, Psychodidae). Cadernos de Saúde Pública, 6:86-89.

COUTO, R. C. S., 1996. Hidrelétricas e Saúde na Amazônia: Um Estudo sobre a Tendência da Malária na Área do lago da Hidrelétrica de Tucuruí, Pará. Tese de Doutorado, Rio de Janeiro: Escola Nacional de Saúde Pública, Fundação Instituto Oswaldo Cruz.

ELETROBRÁS (Centrais Elétricas Brasileiras), 1990. Plano Diretor de Meio Ambiente do Setor Elétrico, 1991/93. Fundamentos, v. 1. Rio de Janeiro: Eletrobrás.

FIGUEIREDO, L. C. de \& RICCI, C. N., 1989. Espécie nova de Mytilopsis conrad 1857, do rio Tocantins, Tucuruí, Pará, Brasil (Molusca Bivalvia, Dreisseridae). Memórias do Instituto Oswaldo Cruz, 84 (Sup. 4):27-33.

GUIMARÃES, A. E. G.; MELLO, R. P.; LOPES, C. M.; ALENCAR, J. \& GENTILE, C., 1997. Prevalência de anophelinos Diptera: Culicidae no crepúsculo vespertino em áreas da usina hidrelétrica de Itaipu no Município de Guaíra, Estado do Paraná, Brasil. Memórias do Instituto Oswaldo Cruz, 92:745-754.

KOIFMAN, S.; FERRAZ, I.; VIANA, T. S.; SILVEIRA, C. L. F.; CARNEIRO, M. T. D.; KOIFMAN, R. J.; SAR- trazidos pela ampliação de recursos energéticos para o país, a industrialização da região norte, o crescimento da oferta de emprego e os benefícios sociais acarretados por este processo; por outro lado, a experiência recente das últimas décadas tem revelado a séria ameaça acarretada sobre a perda da biodiversidade, bem como sobre as condições de vida, saúde, cultura e sobrevivência daquelas populações diretamente afetadas, sobretudo das comunidades indígenas. A reflexão a respeito das conseqüências deste processo e o diálogo com a sociedade civil - incluindo todos os grupos diretamente afetados - deveriam representar novo marco na modificação do processo de geração e transmissão da eletricidade no país, garantindo assim a ampliação do debate acerca de sua implantação e limitação dos danos acarretados à sociedade, como um todo, e às nações indígenas, em particular.

CINELLI, P.; MATTOS, R. C. O. C.; LIMA, J. S.; SILVA, J. O.; MOREIRA, J. C.; FERNANDES, C. \& BULCÃO, A. C., 1998. Cancer cluster among young Indian adults living near power transmission lines in Bom Jesus do Tocantins, Para, Brazil. Cadernos de Saúde Pública, 14(Sup. 3):161-172.

MS (Ministério da Saúde), 2000. Atendimento Integral a População Indígena. Novembro de 2000 <http://funasa.gov.br/ind/ind00.htm>.

MULLEN, R. P., 1991. Xingu, a maior usina hidrelétrica do Brasil e o caso Assurini. In: O Cerco Está se Fechando (R. P. Mullen, org.), pp. 114-129, Rio de Janeiro: Editora Fase/Editora Vozes/Belém: Núcleo de Altos Estudos na Amazônia, Universidade Federal do Pará.

PREECE, A. W.; HAND, J. W.; CLARKE, R. N. \& STEWART, A., 2000. Power frequency electromagnetic fields and health. Where's the evidence. Physics in Medicine and Biology, 45:139-154.

SANTOS, S. C. \& NACKE, A., 1988. Povos indígenas e desenvolvimento hidrelétrico na Amazônia. Revista Brasileira de Ciências Sociais, 8:71-84.

SANTOS, S. C. \& NACKE, A., 1991. O Cerco Está se fechando (R. P. Mullen, org.), pp. 78-88, Rio de Janeiro: Editora Fase/Editora Vozes/Belém: Núcleo de Altos Estudos na Amazônia, Universidade Federal do Pará.

TADEI, W. P., 1994. Proliferação de mosquitos na Hidrelétrica de Tucuruí, Pará. In: A Questão Energética na Amazônia. Avaliação e Perspectivas Sócio Ambientais. Anais do Seminário Internacional, pp. 2-13, Belém: Núcleo de Altos Estudos na Amazônia, Universidade Federal do Pará/Museu Paraense Emílio Goeldi.

VIEIRA FILHO, J. P. B., 1994. Malignant tumors among Gavião Indians. Proximity of electromagnetic fields. Revista da Associação Médica Brasileira, 40:137-139. 